Check for updates

London, UK

Cite this as: $B M J$ 2021;372:n433 http://dx.doi.org/10.1136/bmj.n433 Published: 11 February 2021

\section{Covid-19: Arthritis drug tocilizumab reduces deaths in hospitalised patients, study shows}

\author{
Jacqui Wise
}

The anti-inflammatory treatment, tocilizumab, improves survival for patients hospitalised with covid-19, shortens the time to discharge, and reduces the need for a mechanical ventilator, results from the randomised evaluation of covid-19 therapy (Recovery) trial show. ${ }^{1}$

For every 25 patients treated with tocilizumab, one additional life would be saved, according to the researchers from Oxford University.

The benefits were in addition to those seen with the steroid dexamethasone, which is now standard care following earlier positive results from the Recovery trial.

In November, early results from the Remap-Cap platform trial showed that tocilizumab improved outcomes in critically ill patients in intensive care. ${ }^{2}$ These latest results show that the benefit can also be seen in patients who are less severely ill and are on general wards in the hospital.

Updated guidance will be sent to NHS trusts and clinicians on Monday, recommending the use of tocilizumab for patients hospitalised with covid-19. Health secretary Matt Hancock said, "We are working quickly and closely with colleagues across the health system and sector to ensure every NHS patient who needs this treatment should be able to access it-reducing further pressures on the NHS and potentially saving thousands of lives.”

The study, published as a preprint and not yet peer reviewed, compared 2022 patients randomly allocated to receive tocilizumab and 2094 patients who had usual care. All the patients had evidence of inflammation and required oxygen either with a simple face mask, non-invasive ventilation like continuous positive airway pressure, or mechanical ventilation. Two thirds of the patients were under 70 years and two thirds were male. The majority (82\%) were also taking a systemic steroid such as dexamethasone.

The results showed that 596 of the patients (29\%) in the tocilizumab group died within 28 days compared with 694 (33\%) patients in the usual care group. This is a $14 \%$ reduction in relative mortality (rate ratio $0.86 ; 95 \%$ confidence interval 0.77 to $0.96 ; \mathrm{P}=0.007$ ).

The researchers said that treatment with a combination of dexamethasone plus tocilizumab reduced mortality by about one third for patients who need simple oxygen and nearly one half for those needing mechanical ventilation.

Tocilizumab also increased the probability of discharge alive within 28 days from $47 \%$ to $54 \%$ (rate ratio $1.23,95 \%$ CI 1.12 to $1.34 ; \mathrm{P}<0.0001)$. The treatment produced a five day reduction in hospital stays when used in addition to standard care.

The study also found that for patients who were not on invasive mechanical ventilation when they entered the trial, tocilizumab significantly reduced the chance of progressing to invasive mechanical ventilation or death from $3 \%$ to $33 \%$ (risk ratio $0.85 ; 95 \% \mathrm{CI} 0.788$ to $0.93, \mathrm{P}=0.0005$ ).

Martin Landray, professor of medicine and epidemiology at the Nuffield Department of Population Health, University of Oxford, and joint chief investigator, told a Science Media Centre briefing, "We saw benefits consistently in all groups of patients and these were additive benefits, on top of dexamethasone." He added, "This will change practice in the UK and globally."

Landray said that when they started the Recovery trial they thought it was unlikely that one drug alone would make a difference. He explained that, similarly to when a patient is treated for a heart attack, a combination approach can have a big impact.

The evidence on tocilizumab from previous trials has been inconclusive. But the Recovery trial is four times larger than all the other trials combined and as a result the benefits are now clear cut, Landray said.

Tocilizumab, an intravenous drug used to treat rheumatoid arthritis, targets the cytokine interleukin IL- 6 and blocks its pro-inflammatory effect. Peter Horby, professor of emerging infectious diseases in the Nuffield Department of Medicine, University of Oxford, and joint chief investigator for Recovery, explained, "Dexamethasone is more of a shotgun approach, whereas tocilizumab is a more targeted treatment."

The drug is given as an intravenous infusion over an hour, usually as a one off treatment, and costs around $€_{500}\left(€_{570}\right.$; \$691) per patient. This compares with $€_{5}$ for treatment with dexamethasone.

The Recovery trial has previously announced results showing that hydroxychloroquine,

lopinavir-ritonavir, azithromycin, and convalescent plasma have no benefits for patients hospitalised with covid-19. The trial is continuing to investigate Regeneron's monoclonal antibody cocktail, aspirin, colchicine (an anti-inflammatory used to treat gout), and baricitinib (another anti-inflammatory used to treat rheumatoid arthritis).

Horby PW, Campbell M, Staplin N, et al. Tocilizumab in patients admitted to hospital with covid-19 (Recovery): preliminary results of a randomised, controlled, open-label, platform trial.Medrxiv

doi: 10.1101/2021.02.11.21249258 [Preprint]. 2021. www.medrxiv.org/content/10.1101/2021.02.11.21249258v1.

2 Wise J. Covid-19: Critically ill patients treated with arthritis drug tocilizumab show improved outcomes, researchers report. BM/2020;371:m4530. doi: 10.1136/bmj.m4530 pmid: 33214134 